\title{
EDITORIAL
}

\section{High drug attrition rates-where are we going wrong?}

D rug attrition rates for cancer are much higher than in other therapeutic areas. Only $5 \%$ of agents that have anticancer activity in preclinical development are licensed after demonstrating sufficient efficacy in phase III testing, which is much lower than, for example, $20 \%$ for cardiovascular disease. To compound this issue, many new cancer agents are being withdrawn, suspended or discontinued. Figure 1 illustrates that this trend is extremely prevalent for VEGF inhibitors although less so for drugs targeting Aurora B kinase and some targeted therapies. The reasons for this high attrition rate are complex; however, several articles in this issue provide insights into why this is occurring. In essence, the preclinical strategies to evaluate novel agents are suboptimal, and identifying the correct target using appropriate preclinical models will be critical to prevent further drug failures.

Ian Tannock and coauthors discuss the limitations of preclinical models for drug assessment. A key drawback of animal models is that they do not represent the primary tumors from which they are derived in terms of tumor heterogeneity and the mechanisms of drug resistance. Xenograft models lack the broad molecular transformation events that occur in human tumors. Furthermore, since the stromal component of the tumor is not human the effects of the microenvironment on drug response are often not reflective of the primary tumor. Importantly, the growth rates of human-derived xenografts are considerably more rapid than primary tumors and, as a result, are much more likely to respond to antiproliferative agents. Testing of antiproliferative drugs in animal models might provide a false indication of the potential efficacy of a drug. Also, the immune system in such animal models is compromised, hindering the testing of immunomodulatory agents. Genetically-engineered mouse models circumvent some of these limitations as they are immune competent but they still suffer from having rodent-derived stroma.

Getting the target right is a crucial aspect of drug development. Komlodi-Pasztor and coauthors discuss why mitosis-specific agents-including those that target aurora kinases and polo-like kinases-have limited success in the clinic, especially compared with microtubule-targeting agents (MTAs) that have proved successful despite both classes of agent having the same putative target. A closer examination of the mechanisms of action of MTAs reveals that they exert their effects not on mitosis but predominantly via interphase cellular mechanisms and microtubules, which are present in both mitotic and non-mitotic cells. Most human tumors divide slowly, and so mitosis is rare or absent; therefore, it is an unlikely target for MTAs. The doubling times in humans means that cancer cells multiply at similar rates to bone-marrow cells, explaining why agents that target mitosis also cause neutropenia and myelosuppression. Komlodi-Pasztor et al. state that although mitotic kinases produced disappointing results in the clinic, the drugs were well designed and had a valid target - that is mitosis-but unfortunately their efficacy was at the expense of high toxic effects and the fact that not many of the tumor cells were 'druggable' because the target was transient. However, agents that target mitotic kinases have a lower attrition rate compared with other (non-targeted) cancer drugs (Figure 1), which might be because the trial data are not mature but this is promising nevertheless. These findings emphasize the importance of identifying and validating the target and understanding resistance mechanisms. Interestingly, regarding correct target identification, the HER2-targeting agent trastuzumab may have limited benefit in patients with very high levels of HER2, as indicated by an inferior recurrence-free survival compared with patients who express more moderate levels of the target (Joensuu, H. et al. Ann. Oncol. doi:10.1093/annonc/ mdq710). This might explain why some patients with HER2-positive tumors do not respond to trastuzumab, as this agent might affect other HER family members or compromise downstream signaling effects if HER2 levels are extremely high.

Ebos and Kerbel postulate why the success of antiangiogenic agents in the metastatic setting has not been mirrored in the adjuvant setting. They suggest the nature of disease progression following antiangiogenic therapy might be distinct to that seen with cytotoxics. Evidence from preclinical studies indicates that in certain situations antiangiogenics may increase invasiveness and metastatic potential. In orthotopically implanted mouse tumors, an increase in metastasis and shortened survival was seen when mice were treated with an anti-VEGF therapy before metastasis was induced-thus short-term treatment with antiangiogenic drugs may influence micrometastatic disease, which has critical implications for the preclinical models used in drug development.

It is possible that if VEGF suppression is not sustained this could lead to a 'rebound' effect in terms of tumor growth. This may explain the results from the recent AVANT trial, in which a positive effect on progression-free

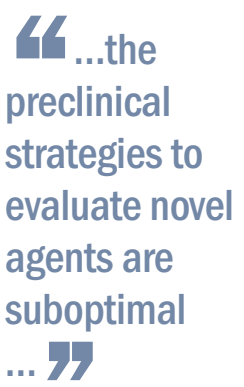

isa Hutchinson is the Chief Editor and Rebecca Kirk is an Associate Editor of Nature Reviews Clinical Oncology.

Competing interests The authors declare no competing interests. 

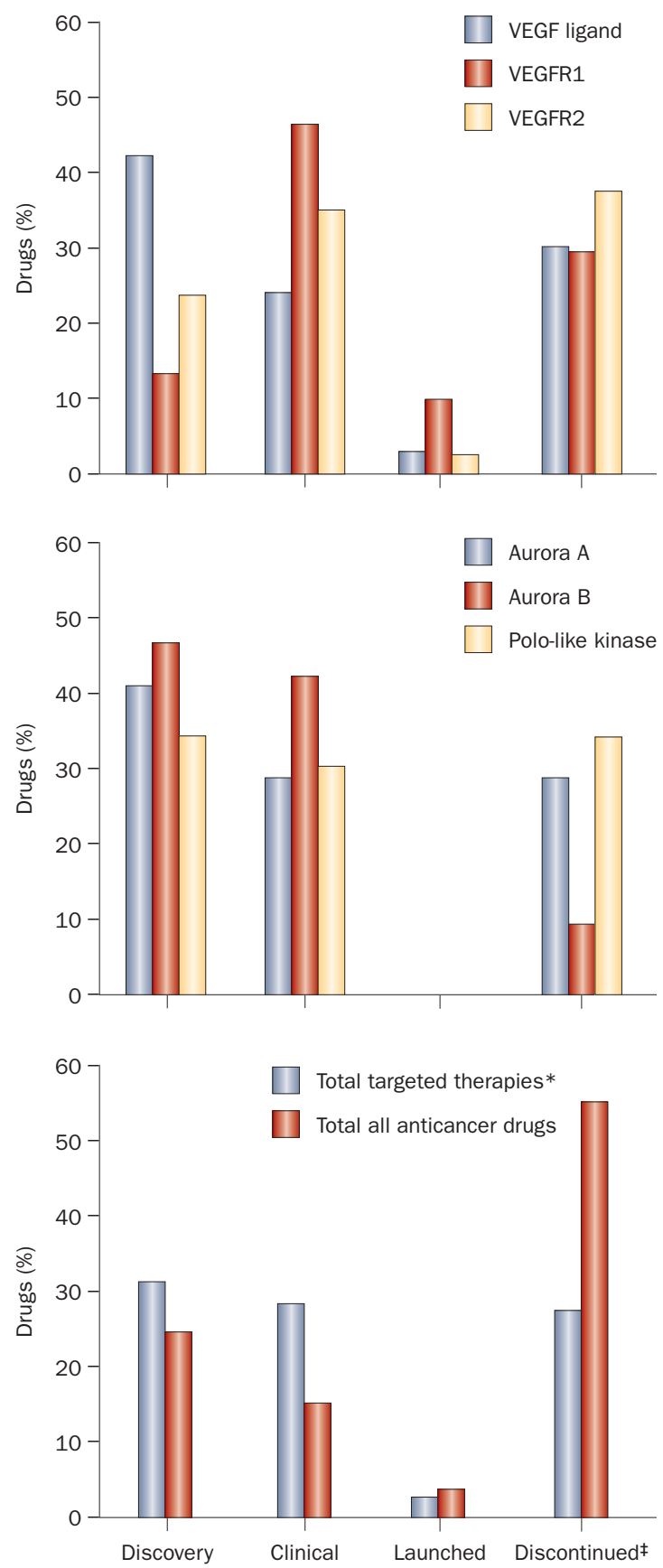

Figure 1 | Anticancer drugs in various stages of development. *Targeted therapies include drugs targeted against Aurora A, Aurora B, BCR-ABL, EGFR, HER2, mTOR, PARP, PI3K, polo-like kinase, VEGF ligand, VEGFR1 and VEGFR2. ₹Discontinued, withdrawn, suspended or no development reported. Analysis of data that were provided courtesy of https://www.thomson-pharma.com.

survival (PFS) initially observed with the bevacizumab and chemotherapy combination in patients with metastatic colorectal cancer was short lived. After a longer assessment period worse overall survival was observed in patients receiving the combination treatment, however, the reason for this has not been established and is likely to be complex. Should 'rebound' effects be tested in all animal models? If the answer is yes, then continued drug dosing and assessment of host responses to therapy (with appropriate controls) would be required before clinical development commences. This would lengthen the time before a drug is tested in the clinic but should ultimately improve drug attrition rates and help identify mechanisms of resistance. The 'rebound' effect also has implications for the end points used in phase II testing, as current end points might not be long enough to detect a relevant clinical change despite initial promising results. Moreover, high levels of drug discontinuation and dose reduction can occur even in clinical situations when antiangiogenic agents have been beneficial, such as the treatment of renal cell carcinoma.

Drugs are usually tested as monotherapy in mouse models rather than in combination with other targeted agents; multiagent testing in animal models would bring us one step closer to representing the clinical situation. Despite the fact that animal model studies have not tested systemic metastatic disease and, therefore, do not include relevant survival-based analysis, VEGF therapy does work in the metastatic setting but not in the adjuvant setting. Clearly, the reasons why antiangiogenic drugs have proved disappointing in the adjuvant setting are more complex than suboptimal animal model testing. As the microenvironment may have a greater influence for antiangiogenics than for cytotoxic agents, drugs that target multiple kinases might prove more successful. This possibility is perhaps evident for the EGFR and VEGF targeted agent sorafenib and might explain the relative success of this drug and other multityrosine kinase inhibitors in terms of drug attrition (Figure 1).

Promising patient responses and PFS outcomes with antiangiogenic agents have not translated to overall survival benefits; thus, the use of PFS as a surrogate for survival is contentious. Crucial questions are why have antiangiogenic agents not delivered when the preclinical data have been so positive, and why have these agents not shown additive efficacy when used with cytotoxics? These questions are unanswered; however, David Kerr comments that the bolus administration of IFL chemotherapy with bevacizumab in the 2004 clinical trial by Hurwitz and coauthors, might have maximized intratumoral cytotoxic drug concentrations resulting in the dramatic benefits seen in this trial. Another possibility is the hypoxia-autophagy effect. In cancer cells, hypoxic conditions increase HIF1 a levels inducing autophagy and angiogenesis, which might explain why rebound effects are seen with antiangiogenic drugs. Unfortunately, there are no predictive biomarkers for bevacizumab; VEGF is not a biomarker of bevacizumab efficacy and it is not known how well VEGF levels correlate with its activity.

For the cancer field to move forward, animal models more representative of the clinical situation should be used, even if this considerably lengthens the time it takes for drugs to reach phase III testing. Of equal importance is the identification and validation of drug molecular targets. Once we get this right we might see lower drug attrition rates.

doi:10.1038/nrclinonc.2011.34 